\title{
Factors related to changes in foot status and foot care in patients with diabetes mellitus participating in an exercise health education program
}

\author{
Kumiko Ozawa ${ }^{1^{*}}$, Toshiko Tomisawa ${ }^{2}$, Sakiko Sakuraba ${ }^{3}$, Mayumi Urushizaka $^{2}$, \\ Maiko Kitajima ${ }^{2}$, Chieko Itaki $^{2}$, Yuka Noto ${ }^{2}$, Kumiko Kawasaki ${ }^{2}$, \\ Hideaki Yamabe ${ }^{2}$, Kyoko Tagami ${ }^{4}$ \\ ${ }^{1}$ Department of Nursing, Hachinohe Gakuin Junior College, Hachinohe, Japan; \\ *Corresponding Author: k-ozawa@jc.hachinohe-u.ac.jp \\ ${ }^{2}$ Hirosaki University Graduate School of Health Sciences, Hirosaki, Japan \\ ${ }^{3}$ Hirosaki University Hospital, Hirosaki, Japan \\ ${ }^{4}$ Aichi Prefectural University, Aichi, Japan
}

Received 28 January 2014; revised 19 February 2014; accepted 25 February 2014

Copyright (c) 2014 Kumiko Ozawa et al. This is an open access article distributed under the Creative Commons Attribution License, which permits unrestricted use, distribution, and reproduction in any medium, provided the original work is properly cited. In accordance of the Creative Commons Attribution License all Copyrights (C) 2014 are reserved for SCIRP and the owner of the intellectual property Kumiko Ozawa et al. All Copyright (C) 2014 are guarded by law and by SCIRP as a guardian.

\section{ABSTRACT}

Purpose: To identify factors related to changes in foot status and foot care in patients with diabetes mellitus participating in an exercise program. Methods: The subjects were 21 patients with Type 2 diabetes mellitus (8 males, 13 females). The mean age of the patients was $62.3 \pm$ 12.5 years old. Foot status, physical measurements and daily activities were monitored and several surveys were performed. Results: Changes in foot status were significantly positively correlated with walking at normal speed $(r=0.54$, $p<0.05)$ and fast speed $(r=0.68, p<0.01)$, and significantly negatively correlated with foot care $(r=-0.51, p<0.05)$. Foot care showed a significant negative correlation with burden of diet therapy $(r=-0.48, p<0.05)$ and a significant positive correlation with the self-efficacy of diet therapy $(r=0.65, p<0.01)$. Conclusions: Subjects who were fast walkers showed greater changes in foot status. Poor foot care behavior is also related to greater changes in foot status. Regular interventions for foot care are important to improve self-care behavior during an exercise program.

\section{KEYWORDS}

Change in Foot Status; Foot Care; Diabetes Mellitus; Exercise

\section{INTRODUCTION}

The world diabetes mellitus population in 2012 was about $371,000,000$, or about $8.3 \%$ of the adult population [1], and the number of patients with diabetes is likely to increase. Leg amputation is common in patients with diabetes, with more than 80,000 of these procedures each year in the USA. Leg amputation reduces QOL and influences self-care behavior such as diet therapy, exercise therapy, and insulin self injection [2].

Delayed or inappropriate treatment of foot lesions in diabetes mellitus can result in infection, gangrene and amputation. Repeated education of patients in foot care can reduce amputation rates by more than 50\% [3] and continuous intervention is more effective than guidance given only once [4]. A foot lesion is associated with substantial morbidity and mortality in diabetic patients and exercise is a protective factor against development of foot lesions [5]. Foot ulcers can be avoided using an exercise program with screening for foot lesions in patients with multiple diabetic complications or a history of foot ulceration [6] and improvement of the shoes of diabetic patients with foot lesions and can result in general improvement of health and mental status [7].

These studies show the importance of nurses supporting foot care and exercise therapy in patients with diabetes. However, there are few studies on the factors related to changes in foot status and foot care during long-term exercise therapy. Therefore, the purpose of this study was to examine these factors in patients with diabetes mellitus participating in an exercise health edu- 
cation program.

\section{MATERIALS AND METHODS}

\subsection{Health Program and Subjects}

A health promotion program for blood sugar control and appropriate exercise was started in June 2007. This study was performed from June 2009 to March 2010. The subjects were 21 patients with Type 2 diabetes mellitus (8 males and 13 females; mean age $62.3 \pm 12.5$ years old). Of these patients, 15 (Group A) participated in the program for more than one year and 6 (Group B) started the program half way through the year (Table 1 ). The health promotion program includes a class meeting one or two times each month. These classes provide information on health education, exercises including walking, stretching and muscle workouts, and group work for self-understanding and understanding of others. Exercise guidance is provided at each class by exercise guidance specialists with experience in this area of more than 20 years.

\subsection{Foot Status}

Foot status was evaluated before and after the exercise program, based on 12 physical items (flare/swelling, cyanosis, cold feeling, drying/crazing, callus/corns, ulcer, horny substance hypertrophy, edema, tinea blanca, dirt, nail abnormality, and transformation) and 5 subjective symptoms (numbness of toes, pain in toes, cold or hot feeling in toes, abnormal feeling, and clamping of the foot).

\subsection{Physical Activity}

Physical activity was evaluated based on the number of steps taken. Subjects wore the Kenz Lifecorder EX for recording daily activity from June to March. A survey and physical measurements of height and weight, body fat percentage, quantity of fat, estimated quantity of bone, quantity of muscle, offal fat level, and walking speed were performed after the exercise health education program.

\subsection{Foot Care Behavior}

The Summary of Diabetes Self-Care Activities (SDSCA) scale [8] was developed to evaluate the selfcare behavior of diabetic patients. The SDSCA consists of 6 subscales of diet, exercise, self monitoring of blood glucose, oral medicine management, foot care, tobacco. Only the 5 items related to foot care were used in this study. Each item was evaluated on a 7-point scale from 0 to 7 , where the score indicates the number of days on which foot care was performed in the previous week. Foot care was also evaluated using an original question- naire consisting of the 10 items shown in Table 2.

\subsection{QOL, Exercise and Diet}

The Elderly Diabetes Impact Scales (EDIS) [9] was developed to evaluate quality of life (QOL) in elderly diabetic patients. The EDIS consists of 6 subscales of burden of symptoms, burden of daily life, burden of diet therapy, burden of drug therapy, satisfaction, and worry, with a total of 37 items. Each item is evaluated on a 4-point scale from 1 to 4 , with a high score indicating a large burden.

A scale has also been developed to evaluate self-efficacy in exercise in patients with diabetes [10]. The scale consists of 8 items, each of which is evaluated on a 2point scale from 2 to 0 , with a high score indicating high self-efficacy. Similarly, a scale was developed to evaluate self-efficacy in diet therapy in patients with diabetes [11]. This scale consists of 2 subscales and 15 items. Each item is evaluated on a 6-point scale from 1 to 6 , with a high score indicating high self-efficacy.

Table 1. Characteristics of the subjects $(n=21)$.

\begin{tabular}{ccc}
\hline Characteristic & Contents & $\mathrm{N}(\%)$ \\
\hline Age (years) & $62.3 \pm 12.5$ & \\
Sex & Male & $8(38.1)$ \\
& Female & $13(61.9)$ \\
Intervention period (years) & 1 (Group A) & $15(71.4)$ \\
& $<1$ (Group B) & $6(28.6)$ \\
Diabetic treatment & Only diet & $6(28.6)$ \\
OMI (kg/m $\left.{ }^{2}\right)$ & Insulin injection & $6(28.6)$ \\
HbA1c $(\%)$ & $24.9 \pm 2.8$ & \\
Mean steps & $6.9 \pm 1.3$ & \\
\hline
\end{tabular}

Table 2. Survey questions on foot care.

\begin{tabular}{|c|c|}
\hline 1 & $\begin{array}{l}\text { Do you look carefully at your feet and toes and between } \\
\text { toes every day? }\end{array}$ \\
\hline 2 & Do you wash the sole and between toes carefully? \\
\hline 3 & $\begin{array}{l}\text { Do you check the water temperature by hand before taking } \\
\text { a bath? }\end{array}$ \\
\hline 4 & Do you avoid wearing shoes with bare feet? \\
\hline 5 & Do you wear shoes and socks that match your feet? \\
\hline 6 & Do you wear clean shoes and socks? \\
\hline 7 & Do you check for small stones before wearing shoes? \\
\hline 8 & Do you use moisturizing cream when your feet are dry? \\
\hline 9 & Do you cut your nails straight and not too deeply? \\
\hline 10 & $\begin{array}{l}\text { Do you talk with a doctor or nurse if you notice a foot } \\
\text { injury or abnormality? }\end{array}$ \\
\hline
\end{tabular}




\subsection{Statistical Analysis}

Data are expressed as means \pm SD. Relationships between items were evaluated using Spearman rank correlation coefficient analysis. Each item was evaluated using the Cronbach $\alpha$ trust coefficient. Data were analyzed using SPSS Statistics v. 18.0 (SPSS, Chicago, IL, USA) and $p<0.05$ was considered to be statistically significant.

\subsection{Ethical Consideration}

This study was approved by the Medical Ethics Committee of Hirosaki University Graduate School of Medicine, Hirosaki, Japan. All subjects gave informed consent.

\section{RESULTS}

\subsection{Characteristics of the Subjects}

The 15 patients in Group A took an average of 10,515 \pm 3035 steps per day from June to November and an average of $9119 \pm 2742$ steps per day from December to March. Measurements of HbA1c before and after the exercise program showed maintenance $(< \pm 0.5 \%)$ in 13 patients and aggravation $(>0.5 \%)$ in 2 patients. No patients had an improvement of HbA1c of $>0.5 \%$.

\subsection{Foot Status}

Data for foot status in the 15 patients in Group A before and after the exercise program are shown in Table 3. Abnormal findings for 5 of the 12 items were found in 6 patients (40.0\%) before the exercise program and in 9 (60\%) by the end of the exercise program. These findings included nail abnormality in 13 subjects (86.6\%), transformation in 13 (86.6\%), and drying/crazing, callus/ corns, and horny substance hypertrophy in 9 subjects (60.0\%) each. Ten items were abnormal in more subjects after the program compared to before the start of the program.

The most common subjective symptom was clamping of the foot, which was present in 7 subjects (33.3\%) before and at the end of program. Numbness and pain in toes were present in 2 subjects (13.3\%) before the exercise program and in $5(33.3 \%)$ at the end of the program.

\subsection{Foot Care}

The number of days of foot care for each item in the SDSCA did not differ significantly before and after the exercise program. A comparison of these results during the program at the end of March also showed no significant difference between Groups A and B. The Cronbach $\alpha$ for foot care was 0.81 and the total score for foot care showed a significant positive correlation with the number
Table 3. Changes in foot status from before to after the exercise program.

\begin{tabular}{|c|c|c|c|}
\hline Symptom type & Symptom & $\begin{array}{l}\text { Before } \\
\mathrm{N}(\%)\end{array}$ & $\begin{array}{l}\text { After } \\
\text { N (\%) }\end{array}$ \\
\hline \multirow[t]{12}{*}{ Physical } & Flare/swelling & $2(13.3)$ & $3(20.0)$ \\
\hline & Cyanosis & $0(0.0)$ & $2(13.3)$ \\
\hline & Cold feeling & $1(0.6)$ & $5(33.3)$ \\
\hline & Drying/crazing & $4(26.6)$ & $9(60.0)$ \\
\hline & Callus/corns & $7(46.6)$ & $9(60.0)$ \\
\hline & Ulcer & $0(0.0)$ & $0(0.0)$ \\
\hline & $\begin{array}{l}\text { Horny substance } \\
\text { hypertrophy }\end{array}$ & $3(20.0)$ & $9(60.0)$ \\
\hline & Edema & $2(13.3)$ & $3(20.0)$ \\
\hline & Tinea blanca & $5(33.3)$ & $7(46.6)$ \\
\hline & Dirt & $2(13.3)$ & $1(0.6)$ \\
\hline & Nail abnormality & $9(60.0)$ & 13 (86.6) \\
\hline & Transformation & $9(60.0)$ & $13(86.6)$ \\
\hline \multirow[t]{5}{*}{ Subjective } & Numbness of toes & $2(13.3)$ & $5(33.3)$ \\
\hline & Pain in toes & $2(13.3)$ & $5(33.3)$ \\
\hline & $\begin{array}{l}\text { Cold or hot feeling } \\
\text { in toes }\end{array}$ & $4(26.6)$ & $4(26.6)$ \\
\hline & Abnormal feeling & $1(0.6)$ & $2(13.3)$ \\
\hline & Clamping of the foot & $7(46.6)$ & $7(46.6)$ \\
\hline
\end{tabular}

of days of foot care $(r=0.64, p<0.01)$. The scores for each item and the total points for foot care did not differ significantly before and after the exercise program, but 9 items had higher scores before the program compared to those after the program. There was no significant difference between Groups A and B.

\subsection{Factors Related to Changes in Foot Status and Foot Care}

Changes in foot status in the 21 subjects from before to after the exercise program showed a significant positive correlation with walking at normal speed $(\mathrm{r}=0.54, p<$ $0.05)$ and walking at fast speed $(\mathrm{r}=0.68, p<0.01)$, and a significant negative correlation with overall foot care scores on the SDSCA $(r=-0.51, p<0.05)$ (Table 4). Foot care showed a significant negative correlation with burden of diet therapy on the EDIS ( $\mathrm{r}=-0.48, p<$ 0.05 ) and a significant positive correlation with the selfefficacy of diet therapy ( $r=0.65, p<0.01$ ) (Figure 1 ). Changes in foot status and foot care showed no significant relationship with age, sex, intervention period, type of diabetic treatment, BMI, HbA1c, average steps, average walking distance, or confidence about exercise.

\section{DISCUSSION}

After the exercise program, more than half of the sub- 
Table 4. Factors related to changes in foot status in correlation analysis $(\mathrm{n}=21)$.

\begin{tabular}{ccc}
\hline Variable & $r$ & $p$ \\
\hline Walking at normal speed & 0.54 & $<0.05$ \\
Walking at fast speed & 0.68 & $<0.01$ \\
Total score for foot care behavior & -0.51 & $<0.05$ \\
\hline
\end{tabular}
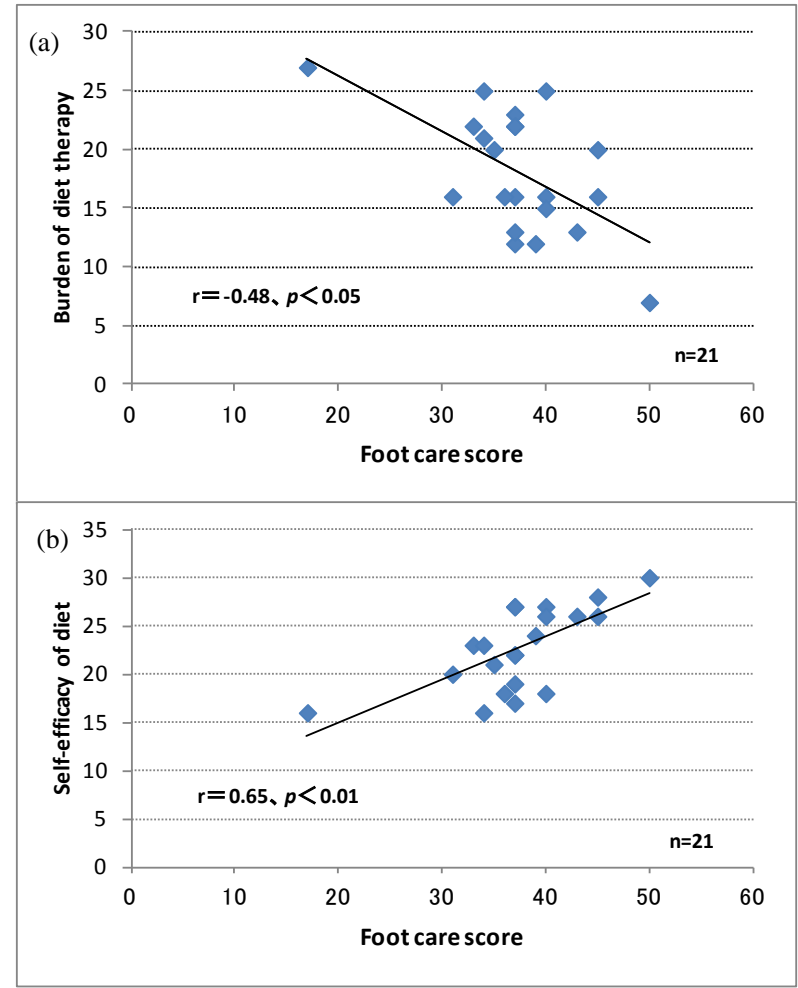

Figure 1. (a) Relationship between foot care and burden of diet therapy. (b) Relationship between foot care and the self-efficacy of diet.

jects had more than five foot abnormalities. These results suggest that exercise may worsen foot lesions in diabetic patients. This may be due to the burden placed on the foot by exercise and wearing of poorly fitted shoes. A seasonal influence may have caused cyanosis, a cold feeling, drying/crazing, and horny substance hypertrophy because the exercise program was held in winter.

More changes in foot status were found in subjects with a fast walking speed. Poor foot care behavior was also related to more changes in foot state. Foot changes are related to cadence and stride length, and these variables can be improved by training, including gait and balance exercises with function-oriented strengthening [12]. Pressure mostly appears in the metatarsal head region in the push-off position and there is a need for appropriate material and structure of shoes to protect the foot from excessive pressure [13]. Therefore, an ideal walking style, cadence and stride length learned in an exercise program and practiced in daily life is likely to reduce the risk of a foot lesion. Also, since improvement of shoes of diabetic patients can result in a better general state of physical and mental health [7], the choice of appropriate shoes is important to maintain self-care behavior in patients with diabetes.

Our results that changes in foot status were not related to BMI are similar to previous findings [14]. The changes in foot status and foot care did not show a significant dependence on physical activity, based on the average number of steps and average walking distance. However, it is important to promote foot care in an exercise program for patients with diabetes who are at high risk for a foot lesion, including identification of patients who do not perform self-care effectively and require intervention to reduce this risk. Continuous intervention is effective [4] and we suggest that this kind of intervention for observation of foot status and foot care is needed before and during an exercise program.

The changes in foot status and foot care did not have a significant relationship with a feeling of confidence during exercise. This may be because the original level of interest in exercise was high in the subjects who participated in the exercise program. In addition, foot care showed a significant negative correlation with the burden of diet therapy and a significant positive correlation with self-efficacy of diet. These results are consistent with previous findings showing that foot care nursing intervention was effective for improving both foot care and diet self-care in patients with diabetes [8]. Foot care showed a strong negative connection with dietary adjustment, which suggests that the burden of thinking about a diet makes it harder to engage in good physical care, such as foot care. In contrast, good foot care behavior led to improved dietary behavior. Regular telephone intervention for patients with diabetes raises recognition of self-efficacy and self-care, increases the frequency of exercise and observation of feet, improves diet and depressive symptoms, and reduces the clinical symptoms of diabetes [15]. Therefore, general and psychological support for blood sugar control and diet therapy may be important for increasing the frequency of foot care.

\section{CONCLUSION}

Subjects who were fast walkers showed more changes in foot status. Foot care showed significant correlations with the burden of diet therapy and the self-efficacy of diet. Therefore, regular intervention for foot care is important to improve self-care behavior in diet therapy during an exercise program.

\section{REFERENCES}

[1] International Diabetes Federation (IDF) (2013) 
http://www.idf.org/diabetesatlas/.

[2] Ragnarson, T.G. and Apelqvist, J. (2000) Health-related quality of life in patients with diabetes mellitus and foot ulcers. Journal of Diabetes and Its Complications, 14, 235-241. http://dx.doi.org/10.1016/S1056-8727(00)00133-1

[3] Marvin, E. and Levin. M.D. (1996) Foot lesions in patients with diabetes mellitus. Endocrinology Metabolism Clinics of North America, 25, 447-462. http://dx.doi.org/10.1016/S0889-8529(05)70333-9

[4] Rijken, P.M., Dekker, J., Lankhorst, G.J. et al. (1999) Podiatric care for diabetic patients with foot problems: An observational study. International Journal of Rehabilitation Research, 22, 181-188. http://dx.doi.org/10.1097/00004356-199909000-00004

[5] Brito-Zurita, O.R., Ortega-López, S., López del CastilloSánchez, D., Vázquez-Téllez, A.R. and Ornelas-Aguirre, J.M. (2013) Ankle-brachial index associated with diabetic foot. Cirugia y Cirujanos, 81, 131-137.

[6] Otterman, N.M., van der Schaaf, M., Busch-Westbroek, T.E., van Schie, C.H. and Nollet, F. (2012) The use and safety of combined resistance and aerobic training in a patient with complications related to Type 2 diabetes. $\mathrm{Di}$ sability and Rehabilitation, 34, 1495-1500. http://dx.doi.org/10.3109/09638288.2011.650312

[7] Davies, S., Gibby, O., Phillips, C. et al. (2000) The health status of diabetic patients receiving orthotic therapy. Quality of Life Research, 9, 233-240. http://dx.doi.org/10.1023/A:1008979825851

[8] Daitoku, M. and Egawa, T. (2004) Evaluation of nursing intervention on foot care behavior in the patients with diabetes mellitus. Journal of Japan Academy of Diabetes
Education and Nursing, 8, 13-24.

[9] Araki, A., Izumo, Y., Inoue, J. et al. (1995) Development of Elderly Diabetes Impact Scales (EDIS) in elderly patients with diabetes mellitus. Nihon Ronen Igakkai Zasshi, 32, 786-796. http://dx.doi.org/10.3143/geriatrics.32.786

[10] Hashimoto, S. Iwasaki, Y. Munakata, T. and Ezawa, I. (1997) A study of perceived confidence in physical exercise, dietary life and health management. The Japanese Journal of Health Behavioral Science, 12, 205-222.

[11] Yasukata, F. (1997) The development of a self-efficacy standard for dietary self-care of patients with diabetes mellitus. Dissertation Doctor, the University of Tokyo, Tokyo.

[12] Allet, L., Armand, S., Aminian, K. et al. (2010) An exercise intervention to improve diabetic patients' gait in a real-life environment. Gait \& Posture, 32, 185-190. http://dx.doi.org/10.1016/j.gaitpost.2010.04.013

[13] Actis, R.L., Ventura, L.B., Smith, K.E. et al. (2006) Numerical simulation of the plantar pressure distribution in the diabetic foot during the push-off stance. Medical \& Biologicall Engineering \& Computing, 44, 653-663. http://dx.doi.org/10.1007/s11517-006-0078-5

[14] Flahr, D. (2010) The effect of nonweight-bearing exercise and protocol adherence on diabetic foot ulcer healing: A pilot study. Ostomy Wound Management, 56, 40-50.

[15] Sacco, W.P., Malone, J.I., Morrison, A.D., Friedman, A. and Wells, K. (2009) Effect of a brief, regular telephone intervention by paraprofessionals for Type 2 diabetes. Journal of Behavioral Medicine, 32, 349-359. http://dx.doi.org/10.1007/s10865-009-9209-4 